\title{
Uniform Difference Scheme on the Singularly Perturbed System
}

\author{
Ilhame G. Amiraliyeva \\ Department of Mathematics, Faculty of Science, Sinop University, Sinop, Turkey \\ Email: ailhame@gmail.com
}

Received July 12, 2012; revised August 12, 2012; accepted August 19, 2012

\begin{abstract}
This paper is concerned with the numerical solution for singular perturbation system of two coupled second ordinary differential equations with initial and boundary conditions, respectively. Fitted finite difference scheme on a uniform mesh, whose solution converges pointwise independently of the singular perturbation parameter is constructed and analyzed.
\end{abstract}

Keywords: Singular Perturbation; Linear System; Difference Scheme; Uniform Convergence

\section{Introduction}

We consider the following singularly perturbed initial/ boundary value problem for the linear system of ordinary differential equations in the interval $[0,1]$ :

$$
\begin{gathered}
L_{1} u:=\varepsilon u^{\prime \prime}(x)+a_{1}(x) u^{\prime}(x)+b_{1}(x) u(x) \\
=c_{1}(x) v(x)+f_{1}(x), \\
L_{2} u:=-\varepsilon v^{\prime \prime}(x)+a_{2}(x) v(x) \\
=\varepsilon^{\mu} c_{2}(x) u(x)+f_{2}(x), \\
u(0)=A_{1}, u^{\prime}(0)=\frac{B_{1}}{\varepsilon} \\
v(0)=A_{2}, v(1)=B_{2},
\end{gathered}
$$

where $\varepsilon>0$ is a small parameter, $\mu \geq 0, A_{1}, A_{2}, B_{1}$, $B_{2}$, are given constants. The functions $a_{i}(x) \geq \alpha_{i}>0$, $c_{i}(x), f_{i}(x)(i=1,2), b_{1}(x)$ are given functions satisfying certain regularity conditions which are specified whenever necessary.

The above type initial/boundary value problems arise in many areas of mechanics and physics [1,2].

Differential equations with a small parameter $\varepsilon$ multiplying the highest order derivative terms are said to be singularly perturbed and normally boundary layers occur in their solutions. The numerical analysis of singular perturbation cases has always been far from trivial because of the boundary layer behavior of the solution. Such problems undergo rapid changes within very thin layers near the boundary or inside the problem domain. It is well known that standard numerical methods for solving such problems are unstable and fail to give accurate results when the perturbation parameter $\varepsilon$ is small. Therefore, it is important to develop suitable numerical methods to these problems, whose accuracy does not depend on the parameter value $\varepsilon$, i.e. methods that are $\varepsilon$-uniformly convergent. These include fitted finite difference methods, finite element methods using special elements such as exponential elements, and methods which use a priori refined or special non-uniform grids which condense in the boundary layers in a special manner. The various approaches to the design and analysis of appropriate numerical methods for singularly perturbed differential equations can be found in [3-8] (see also references cited in them).

In this present paper, we analyze the numerical solution of the initial/boundary problems (1)-(4). The numerical method presented here comprises a fitted difference scheme on an uniform mesh. Fitted operator method is widly used to construct and analyse uniform difference methods, especially for a linear differential problems (see, e.g., [4-7]). In the Section 2, we state some important properties of the exact solution. The derivation of the difference scheme and uniform convergence analysis have been given in Section 3. Uniform convergence is proved in the discrete maximum norm. The approach to the construction of the discrete problem and the error analysis for the approximate solution are similar to those in $[8,9]$.

Difference schemes for singularly perturbed systems with another type of initial/boundary conditions was investigated in [9-14].

Throughout the paper, $\mathrm{C}$ will denote a generic positive constant independent of $\varepsilon$ and of the mesh parameter. 


\section{Analytical Results}

Here we give useful asymptotic estimates of the exact solution of (1.1)-(1.4), that are needed in later sections.

Lemma 2.1 Under the

$$
\rho=\varepsilon^{\mu}\left(\alpha_{1} \alpha_{2}\right)^{-1}\left\|c_{1}\right\|_{\infty}\left\|c_{2}\right\|_{\infty} \exp \left(\|b\|_{\infty} \alpha_{1}^{-1}\right)<1
$$

the problem (1.1)-(1.4) has a unique solution, which satisfies

$$
\begin{gathered}
\|u\|_{\infty} \leq C, \\
\left|u^{\prime}(x)\right| \leq C\left(1+\frac{1}{\varepsilon} \exp \left(-\alpha_{1} x / \varepsilon\right)\right), 0 \leq x \leq l, \\
\|v\|_{\infty} \leq C \\
\left|v^{\prime}(x)\right| \leq C\left[1+\varepsilon^{-\frac{1}{2}} \exp \left(-x \sqrt{a_{2}(0) / \varepsilon}\right)\right. \\
\left.+\varepsilon^{-\frac{1}{2}} \exp \left(-(1-x) \sqrt{a_{2}(1) / \varepsilon}\right)\right],
\end{gathered}
$$

where $\|g\|_{\infty}=\max _{[0,1]}|g(x)|$ for any continuous function $g(x)$.

Proof. Consider the iterative process

$$
\left\{\begin{array}{l}
L_{1} u^{(n)}=f_{1}(x)+c_{1}(x) v^{(n-1)}, \\
L_{2} v^{(n)}=f_{2}(x)+\varepsilon^{\mu} c_{2}(x) u^{(n)}, \\
u^{(n)}(0)=A_{1}, u^{(n)}(0)=\frac{B_{1}}{\varepsilon}, \\
v^{(n)}(0)=A_{2}, v^{(n)}(1)=B_{2},
\end{array}\right.
$$

where $v^{(0)}(x) \in C[0,1]$ is an arbitrary function.

First we prove that for the solution of initial-value problem of the type

$$
\begin{gathered}
\varepsilon u^{\prime \prime}(x)+a(x) u^{\prime}(x)+b(x) u(x)=F(x), \\
u(0)=A, u^{\prime}(0)=\frac{B}{\varepsilon}
\end{gathered}
$$

the following estimates hold

$$
\begin{aligned}
\|u(x)\|_{\infty} \leq & \exp \left(\|b\|_{\infty} \alpha^{-1}\right)\left(|A|+\alpha^{-1}|B|+\alpha^{-1}\|F\|_{\infty}\right), \\
\left|u^{\prime}(x)\right| \leq & \exp \left(\|b\|_{\infty} \alpha^{-1}\right)\|b\|_{\infty}|A| \\
& +\alpha^{-1}\|b\|_{\infty} \exp \left(\|b\|_{\infty} \alpha^{-1}\right)\|B\| \\
& +\frac{1}{\varepsilon} \exp (-\alpha x / \varepsilon)|B| \\
& +\alpha^{-1}\left[\alpha^{-1}\|b\|_{\infty} \exp \left(\|b\|_{\infty} \alpha^{-1}\right)+1\right]\|F\|_{\infty} .
\end{aligned}
$$

To prove (2.7), after some manipulations we have

$$
\begin{aligned}
|u(x)| & \leq|A|+|B| \alpha^{-1}+\alpha^{-1}\|b\|_{\infty} \int_{0}^{x}|u(\eta)| \mathrm{d} \eta+\alpha^{-1}\|F\|_{\infty} \\
& =|A|+\left(|B|+\|F\|_{\infty}\right) \alpha^{-1}+\alpha^{-1}\|b\|_{\infty} \int_{0}^{x}|u(\eta)| \mathrm{d} \eta,
\end{aligned}
$$

hence

$$
|u(x)| \leq A^{*}+B^{*} \int_{0}^{x}|u(\eta)| \mathrm{d} \eta
$$

with

$$
\begin{gathered}
A^{*}=|A|+\left(|B|+\|F\|_{\infty}\right) \alpha^{-1}, \\
B^{*}=\alpha^{-1}\|b\|_{\infty} .
\end{gathered}
$$

From here by virtue of integral inequality it follows that

$$
|u(x)| \leq A^{*} e^{B^{*}}
$$

which leads to (2.7). Now we prove (2.8). Clearly

$$
\left|u^{\prime}(x)\right| \leq \frac{|B|}{\varepsilon} \exp (-\alpha x / \varepsilon)+\left(\|u\|_{\infty}\|b\|_{\infty}+\|F\|_{\infty}\right) \alpha^{-1} .
$$

Then by using (2.7) we get

$$
\begin{aligned}
\left|u^{\prime}(x)\right| \leq & \frac{|B|}{\varepsilon} \exp (-\alpha x / \varepsilon)+\alpha^{-1}\|b\|_{\infty}\left(|A| \exp \left(\|b\|_{\infty} \alpha^{-1}\right)\right. \\
& \left.+\|F\|_{\infty} \alpha^{-1} \exp \left(\|b\|_{\infty} \alpha^{-1}\right)+|B| \exp \left(\|b\|_{\infty} \alpha^{-1}\right)\right) \\
& +\alpha^{-1}\|b\|_{\infty}|A| \exp \left(\|b\|_{\infty} \alpha^{-1}\right) \\
& +\left(\exp (-\alpha x / \varepsilon) / \varepsilon+\alpha^{-2}\|b\|_{\infty} \exp \left(\|b\|_{\infty} \alpha^{-1}\right)\right)|B| \\
& +\left(\alpha^{-1}\|b\|_{\infty} \exp \left(\|b\|_{\infty} \alpha^{-1}\right)+1\right)\|F\|_{\infty} \alpha^{-1},
\end{aligned}
$$

which arrive at (2.8).

Further, note that, by virtue of maximum principle the problem of the form

$$
\begin{gathered}
L_{2} v=F(x), F(x) \in C[0,1] \\
v(0)=A, v(1)=B
\end{gathered}
$$

admits the estimate

$$
|v| \leq|A|+|B|+\alpha^{-1}\|F\|_{\infty} .
$$

Denoting

$$
\begin{aligned}
& \delta^{(n)}(x)=u^{(n)}(x)-u^{(n-1)}(x), \\
& \theta^{(n)}(x)=v^{(n)}(x)-v^{(n-1)}(x)
\end{aligned}
$$

from (1.1)-(1.4) and (2.6) we have

$$
\left\{\begin{array}{l}
L_{1} \delta^{(n)}=c_{1} \theta^{(n-1)}, \\
L_{2} \theta^{(n)}=\varepsilon^{\mu} c_{2} \delta^{(n-1)}, \\
\delta^{(n)}(0)=\theta^{\prime}(0)=0, \\
\theta^{(n)}(0)=\theta^{(n)}(1)=0 .
\end{array}\right.
$$


Next, applying here (2.7), (2.8), (2.9) we arrive at $\left\|\delta^{(n)}\right\|_{\infty} \leq \alpha_{1}^{-1} \exp \left(\|b\|_{\infty} \alpha^{-1}\right)\left\|c_{1}\right\|_{\infty}\left\|\theta^{(n-1)}\right\|_{\infty}$, $\left\|\delta^{(n)}\right\|_{\infty} \leq \alpha_{1}^{-1}\left[\alpha_{1}^{-1}\|b\|_{\infty} \exp \left(\|b\|_{\infty} \alpha^{-1}\right)+1\right]\left\|c_{1}\right\|_{\infty}\left\|\theta^{n-1}\right\|_{\infty}$, $\left\|\theta^{(n)}\right\|_{\infty} \leq \frac{\varepsilon^{\mu}}{\alpha_{2}}\left\|c_{2}\right\|_{\infty}\left\|\delta^{(n-1)}\right\|_{\infty}$.

Therefore

$$
\begin{aligned}
& \left\|\delta^{(n)}\right\|_{\infty} \leq \rho_{1}\left\|\theta^{(n-1)}\right\|_{\infty}, \\
& \left\|\delta^{(n)}\right\|_{\infty} \leq \rho_{3}\left\|\theta^{(n-1)}\right\|_{\infty}, \\
& \left\|\theta^{(n)}\right\|_{\infty} \leq \rho_{2}\left\|\delta^{(n)}\right\|_{\infty},
\end{aligned}
$$

with

$$
\begin{aligned}
& \rho_{1}=\alpha_{1}^{-1} \exp \left(\|b\|_{\infty} \alpha^{-1}\right)\left\|c_{1}\right\|_{\infty}, \\
& \rho_{2}=\alpha_{2}^{-1} \varepsilon^{\mu}\left\|c_{2}\right\|_{\infty}, \\
& \rho_{3}=\alpha_{1}^{-1}\left[\alpha_{1}^{-1}\|b\|_{\infty} \exp \left(\|b\|_{\infty} \alpha^{-1}\right)+1\right]\left\|c_{1}\right\|_{\infty} .
\end{aligned}
$$

From (2.10) we have

$$
\begin{gathered}
\left\|\theta^{(n)}\right\|_{\infty} \leq \rho_{1} \rho_{2}\left\|\theta^{(n-1)}\right\|_{\infty} \leq\left(\rho_{1} \rho_{2}\right)^{n-1}\left\|\theta^{(1)}\right\|_{\infty}, \\
\left\|\delta^{(n)}\right\|_{\infty} \leq\left(\rho_{1} \rho_{2}\right)^{n-2} \rho_{1}\left\|\theta^{(1)}\right\|_{\infty}, \\
\left\|\delta^{(n)}\right\|_{\infty} \leq \rho_{3}\left(\rho_{1} \rho_{2}\right)^{n-2}\left\|\theta^{(1)}\right\|_{\infty} .
\end{gathered}
$$

From (2.12), (2.13), (2.14) follows that the sequences $\left\{u^{(n)}\right\},\left\{u^{(n)}\right\},\left\{v^{(n)}\right\}$ uniformly converges on $x \in[0,1]$ for $n \rightarrow \infty$. Replacing (2.6) by appropriate system of integral equations we conclude that for $n \rightarrow \infty$ the limit functions are the solution of (1.1)-(1.4).

Now the using (2.7) and (2.8) with the function $F(x)=c_{1}(x) v(x)+f_{1}(x)$ yield the following stability bounds

$$
\begin{aligned}
\|u\|_{\infty} \leq & \exp \left(\|b\|_{\infty} \alpha^{-1}\right)\left|A_{1}\right|+\alpha_{1}^{-1} \exp \left(\|b\|_{\infty} \alpha^{-1}\right)\left|B_{1}\right| \\
& +\alpha_{1}^{-1}\left\|c_{1}\right\|_{\infty} \exp \left(\|b\|_{\infty} \alpha^{-1}\right)\|v\|_{\infty} \\
& +\frac{1}{\alpha_{1}} \exp \left(\|b\|_{\infty} \alpha_{1}^{-1}\right)\left\|f_{1}\right\|_{\infty} \\
\left|u^{\prime}\right| \leq & \|b\|_{\infty} \exp \left(\|b\|_{\infty} \alpha^{-1}\right)\left|A_{1}\right| \\
& +\alpha_{1}^{-1}\|b\|_{\infty} \exp \left(\|b\|_{\infty} \alpha_{1}^{-1}\right)\left|B_{1}\right| \\
& +\frac{1}{\varepsilon} \exp \left(-\alpha_{1} x / \varepsilon\right)\left|B_{1}\right| \\
& +\alpha_{1}^{-1}\left[\alpha_{1}^{-1}\|b\|_{\infty} \exp \left(\|b\|_{\infty} \alpha_{1}^{-1}\right)+1\right]\left\|f_{1}\right\|_{\infty} \\
& +\alpha_{1}^{-1}\left[\|b\|_{\infty} \alpha_{1}^{-1} \exp \left(\|b\|_{\infty} \alpha_{1}^{-1}\right)+1\right]\left\|c_{1}\right\|_{\infty}\|v\|_{\infty} .
\end{aligned}
$$

Next from (2.9), with $F(x)=\varepsilon^{\mu} c_{2}(x) u(x)+f_{2}(x)$ it follows that

$$
\|v\|_{\infty} \leq\left|A_{2}\right|+\left|B_{2}\right|+\varepsilon^{\mu} \alpha_{2}^{-1}\left\|c_{2}\right\|_{\infty}\|u\|_{\infty}+\alpha_{2}^{-1}\left\|f_{2}\right\|_{\infty} .
$$

From (2.13) and (2.15) obviously

$$
\begin{aligned}
\|u\|_{\infty} \leq & (1-\rho)^{-1}\left\{\exp \left(\|b\|_{\infty} \alpha_{1}^{-1}\right)\left|A_{1}\right|\right. \\
& +\alpha_{1}^{-1} \exp \left(\|b\|_{\infty} \alpha_{1}^{-1}\right)\left|B_{1}\right|+\alpha_{1}^{-1} \exp \left(\|b\|_{\infty} \alpha^{-1}\right)\left\|f_{1}\right\|_{\infty} \\
& \left.+\alpha_{1}^{-1}\left\|c_{1}\right\|_{\infty} \exp \left(\|b\|_{\infty} \alpha_{1}^{-1}\right)\left(\left|A_{2}\right|+\left|B_{2}\right|+\alpha_{2}^{-1}\left\|f_{2}\right\|_{\infty}\right)\right\} \\
\|v\|_{\infty} \leq & (1-\rho)^{-1}\left\{\left|A_{2}\right|+\left|B_{2}\right|+\alpha_{2}^{-1}\left\|f_{2}\right\|_{\infty}+\varepsilon^{\mu_{2}} \alpha_{2}\left\|c_{2}\right\|_{\infty}\right. \\
& \times\left(\exp \left(\|b\|_{\infty} \alpha_{1}^{-1}\right)\left|A_{1}\right|+\alpha_{1}^{-1} \exp \left(\|b\|_{\infty} \alpha_{1}^{-1}\right)\left|B_{1}\right|\right. \\
& \left.\left.+\alpha_{1}^{-1} \exp \left(\|b\|_{\infty} \alpha_{1}^{-1}\right)\left\|f_{1}\right\|_{\infty}\right)\right\} .
\end{aligned}
$$

Using the last relation in (2.14) we obtain $\left|u^{\prime}\right| \leq\left|b \|_{\infty} \exp \left(\|b\|_{\infty} \alpha_{1}^{-1}\right)\right| A_{1} \mid$

$$
\begin{aligned}
& +\alpha_{1}^{-1}\|b\|_{\infty} \exp \left(\|b\|_{\infty} \alpha^{-1}\right)\left|B_{1}\right|+\frac{1}{\varepsilon} e^{-\frac{\alpha_{1} x}{\varepsilon}}\left|B_{1}\right| \\
& +\alpha_{1}^{-1}\left[\alpha_{1}^{-1}\|b\|_{\infty} \exp \left(\|b\|_{\infty} \alpha_{1}^{-1}\right)+1\right]\left\|f_{1}\right\|_{\infty} \\
& +\alpha_{1}^{-1}\left[\|b\|_{\infty} \alpha_{1}^{-1} \exp \left(\|b\|_{\infty} \alpha_{1}^{-1}\right)+1\right] \\
& \times\left\|c_{1}\right\|_{\infty}(1-\rho)^{-1}\left\{\left|A_{2}\right|+\left|B_{2}\right|+\alpha_{2}^{-1}\left\|f_{2}\right\|_{\infty}\right. \\
& +\varepsilon^{\mu} \alpha_{2}^{-1}\left\|c_{2}\right\|_{\infty} \exp \left(\|b\|_{\infty} \alpha_{1}^{-1}\right)\left|A_{1}\right| \\
& \left.+\alpha_{1}^{-1} \exp \left(\|b\|_{\infty} \alpha_{1}^{-1}\right)\left|B_{1}\right|+\alpha_{1}^{-1} \exp \left(\|b\|_{\infty} \alpha_{1}^{-1}\right)\left\|f_{1}\right\|_{\infty}\right\} .
\end{aligned}
$$

The last three inequalities show the validity of (2.2)(2.4). Now we prove (2.5). Since

$$
\begin{aligned}
\left|\left(L_{2} v\right)\right| \leq & \varepsilon^{\mu}\left|c_{2}^{\prime}(x)\right||u(x)| \\
& +\varepsilon^{\mu}\left|c_{2}(x)\right|\left|u^{\prime}(x)\right|+\left|f_{2}(x)\right| \leq C,
\end{aligned}
$$

which leads to (2.5), which completes the proof.

\section{The Difference Scheme and Convergence}

Now we construct the difference scheme and investigate it. In what follows, we denote by $\omega$ the uniform mesh in $[0,1]$ :

$$
\omega=\left\{x_{i}=i h, i=1,2, \cdots, N-1, h=1 / N\right\}
$$

and $\bar{\omega}=\omega \cup\{x=0, l\}$. Before describing our numerical method, we introduce some notation for the mesh functions. For any mesh function $g(x)$, we use

$$
\begin{aligned}
& g_{i}=g\left(x_{i}\right), \\
& g_{\bar{x}, i}=\left(g_{i}-g_{i-1}\right) / h, \\
& g_{x, i}=\left(g_{i+1}-g_{i}\right) / h, \\
& g_{<, i}=\left(g_{i+1}-g_{i-1}\right) /(2 h), \\
& g_{\bar{x} x, i}=\left(g_{i+1}-2 g_{i}+g_{i-1}\right) / h^{2}, \\
& \|g\|_{\infty} \equiv\|g\|_{\infty, d}=\max _{0 \leq i \leq N}\left|g_{i}\right| .
\end{aligned}
$$


On $\bar{\omega}$ we propose the following difference scheme for approximating (1.1)-(1.4):

$$
\begin{gathered}
\mathcal{L}_{1}^{h} U_{i}:=\varepsilon \sigma_{i}^{(1)} U_{\bar{x} x, i}+a_{1 i} U_{\text {o }}+b_{i} U_{i}=c_{1 i} V_{i}+f_{1 i}, \\
\mathcal{L}_{2}^{h} V_{i}:=-\varepsilon \sigma_{i}^{(2)} V_{\bar{x} x, i}+a_{2 i} V_{i}=\varepsilon^{\mu} c_{2 i} U_{i}+f_{2 i}, \\
U_{0}=A_{1}, \\
U_{x, 0}=\left[1+a_{1,0} \frac{h}{\varepsilon} \sigma_{1,0}^{(2)}\right]^{-1}\left(\varepsilon^{-1} B_{1}-b_{0} \frac{h}{\varepsilon} \sigma_{1,0}^{(2)}+\frac{h}{\varepsilon} \sigma_{1,0}^{(2)} f_{1,0}\right), \\
V_{0}=A_{2}, V_{N}=B_{2},
\end{gathered}
$$

and

$$
\begin{aligned}
& \sigma_{i}^{(1)}=\frac{a_{1 i} h}{2 \varepsilon}\left(\sigma_{1 i}^{(2)}+\sigma_{1 i}^{(1)}\right)+1=\frac{h}{2 \varepsilon} a_{1 i} \operatorname{coth}\left(\frac{a_{1 i} h}{2 \varepsilon}\right), \\
& \sigma_{1 i}^{(1)}=\frac{\varepsilon}{a_{1 i} h}-\left(\exp \left(\frac{a_{1 i} h}{\varepsilon}\right)-1\right)^{-1}, \\
& \sigma_{1 i}^{(2)}=\left(1-\exp \left(-\frac{a_{1 i} h}{\varepsilon}\right)\right)^{-1}-\frac{\varepsilon}{a_{1 i} h}, \\
& \sigma_{i}^{(2)}=\frac{h^{2} a_{2 i}}{4 \varepsilon_{\sinh ^{2}}\left(h \sqrt{\frac{a_{2 i}}{2 \varepsilon}}\right)} .
\end{aligned}
$$

For solving of the (3.1)-(3.4) we give the following iterative procedure:

$$
\begin{gathered}
\mathcal{L}_{1}^{h} U_{i}^{(n)}=c_{1 i} V_{i}^{(n-1)}+f_{1 i}, \\
\mathcal{L}_{2}^{h} V_{i}^{(n)}=\varepsilon^{\mu} c_{2 i} U_{i}^{(n)}+f_{2 i}, \\
U_{0}^{(n)}=A_{1}, \\
U_{x, 0}^{(n)}=\left[1+\frac{a_{1,0} h}{\varepsilon} \sigma_{1,0}^{(2)}\right]^{-1}\left(\varepsilon^{-1} B_{1}-b_{0},{ }_{\varepsilon}^{A_{1} h} \sigma_{1,0}^{(2)}+f_{1,0} \frac{h}{\varepsilon} \sigma_{1,0}^{(2)}\right) \\
V_{0}^{(n)}=A_{2}, V_{N}^{(n)}=B_{2},
\end{gathered}
$$

where $V_{i}^{(0)}$ is arbitrary.

\section{Lemma 3.1}

$$
\begin{gathered}
\left\|U^{(n)}-U\right\|_{\infty, d} \leq \frac{\rho_{1} \rho^{n-1}}{1-\rho}\left\|V^{(1)}-V^{(0)}\right\|_{\infty, d}, \\
\left\|V^{(n)}-V\right\|_{\infty, d} \leq \frac{\rho^{n-1}}{1-\rho}\left\|V^{(1)}-V^{(0)}\right\|_{\infty, d},
\end{gathered}
$$

where $\|\cdot\|_{\infty, d}$ implies the discrete maximum norm on $\omega_{h}$; $\rho$ and $\rho_{1}$ are defined by (2.1) and (2.11) appropriately.

Proof. Denoting $\delta_{i}^{(n)}=U_{i}^{(n)}-U_{i-1}^{(n-1)}, \theta_{i}^{(n)}=V_{i}^{(n)}-V_{i-1}^{(n-1)}$ we will have

$$
\begin{gathered}
\mathcal{L}_{1}^{h} \delta_{1}^{(n)}=c_{1 i} \theta^{(n-1)}, \\
\mathcal{L}_{2}^{h} \theta_{i}^{(n)}=\varepsilon^{\mu} c_{2 i} \delta_{i}^{(n)}, \\
\delta_{0}^{(n)}=\delta_{x, 0}^{(n)}=0, \\
\theta_{0}^{(n)}=\theta_{N}^{(n)}=0 .
\end{gathered}
$$

From (3.7)-(3.10), it is not difficult to get

$$
\left|\delta_{i}^{(n)}\right| \leq \gamma h \sum_{i=1}^{j}\left|c_{1 i}\right|\left|\theta_{i}^{(n-1)}\right|,
$$

where

$$
\gamma=4 \alpha^{-1} \exp \left(4 \alpha^{-1}\|b\|_{\infty}\right)
$$

and thereby

$$
\begin{aligned}
\left\|\delta^{(n)}\right\|_{\infty, d} & \leq \gamma j h\left\|c_{1}\right\|_{\infty, d}\left\|\theta^{(n-1)}\right\|_{\infty, d} \\
& \leq \gamma\left\|C_{1}\right\|_{\infty, d}\left\|\theta^{(n-1)}\right\|_{\infty, d}=\rho_{1}\left\|\theta^{(n-1)}\right\|_{\infty, d} .
\end{aligned}
$$

In similar manner we also obtain

$$
\left\|\theta^{(n)}\right\|_{\infty, d} \leq \frac{\varepsilon^{\mu}}{\alpha_{2}}\left\|c_{2}\right\|_{\infty, d}\left\|\delta^{(n)}\right\|_{\infty, d}=\rho_{2}\left\|\delta^{(n)}\right\|_{\infty, d} .
$$

Hence,

$$
\left\|\theta^{(n)}\right\|_{\infty, d} \leq \rho\left\|\theta^{(n-1)}\right\|_{\infty, d}, n=1,2, \cdots
$$

Consequently,

$$
\begin{gathered}
\left\|\theta^{(n)}\right\|_{\infty, d} \leq \rho^{n-1}\left\|\theta^{(1)}\right\|_{\infty, d}, \\
\left\|\delta^{(n)}\right\|_{\infty, d} \leq \rho_{1} \rho^{n-2}\left\|\theta^{(1)}\right\|_{\infty, d} .
\end{gathered}
$$

From this follows that the $\delta^{(n)}, \theta^{(n)} \rightarrow 0$ to $n \rightarrow \infty$, hence the sequences $\left\{U_{i}\right\},\left\{V_{i}\right\}$ are the Cauchy sequences and convergent: $\lim _{n \rightarrow \infty} U_{i}^{(n)}=U_{i}$ and $\lim _{n \rightarrow \infty} V_{i}^{(n)}=V_{i}$. The limit functions $U_{i}, V_{i}$ will be solution of scheme (3.1)-(3.4).

Now we prove (3.5), (3.6). We have

$$
\begin{aligned}
& \left\|U^{(n+m)}-U^{(n)}\right\|_{\infty, d} \\
\leq & \left\|U^{(n+m)}-U^{(n+m-1)}\right\|_{\infty, d}+\left\|U^{(n+m-1)}-U^{(n+m-2)}\right\|_{\infty, d} \\
& +\ldots+\left\|U^{(n+1)}-U^{(n)}\right\|_{\infty, d} \\
\leq & \rho_{1}\left(\rho^{n+m-2}+\rho^{n+m-3}+\ldots+\rho^{n-1}\right)\left\|V^{(1)}-V^{(0)}\right\|_{\infty, d} \\
= & \rho^{n-1} \rho_{1}\left(\rho^{m-1}+\rho^{m-2}+\ldots+1\right)\left\|V^{(1)}-V^{(0)}\right\|_{\infty, d} \\
= & \rho_{1} \rho^{n-1} \frac{1-\rho^{m}}{1-\rho}\left\|V^{(1)}-V^{(0)}\right\|_{\infty, d} \\
\leq & \frac{\rho^{n-1}}{1-\rho}\left\|V^{(1)}-V^{(0)}\right\|_{\infty, d} .
\end{aligned}
$$


The limit case for $m \rightarrow \infty$ leads to (3.5). The inequality (3.6) is being proved analoguosly.

Lemma 3.2 The solution of the difference problem (3.1)-(3.4) satisfies

$$
\begin{aligned}
\|U\|_{\infty, d} \leq & (1-\rho)^{-1} \gamma\left\{\varepsilon \sigma_{*}\left|U_{x, 0}\right|+h \sum_{i=1}^{N}\left|f_{1 i}\right|\right. \\
& \left.+\left\|c_{1}\right\|_{\infty, d}\left(\left|A_{1}\right|+\left|B_{1}\right|+\frac{\| f_{2} \mid}{\alpha_{2}}\right)\right\} \\
\|V\|_{\infty, d} \leq & (1-\rho)^{-1}\left\{\left|A_{2}\right|+\left|B_{2}\right|+\alpha_{2}^{-1}\left\|f_{2}\right\|_{\infty, d}\right. \\
& \left.+\alpha_{2}^{-1} \varepsilon^{\mu} \gamma\left\|c_{2}\right\|_{\infty, d}\left(\varepsilon \sigma_{*}\left|U_{N}\right|+h \sum_{i=1}^{N}\left|f_{1 i}\right|\right)\right\} .
\end{aligned}
$$

where $\sigma_{*}=\frac{h \alpha_{1}}{2 \varepsilon} \operatorname{coth}\left(\frac{h \alpha_{1}}{2 \varepsilon}\right), \quad p=1,2, \cdots, N-1$.

Proof. Using the estimates for the difference equations

$$
\mathcal{L}_{1}^{h} U_{i}=F_{i}
$$

and

$$
\mathcal{L}_{2}^{h} V_{i}=G_{i}
$$

with conditions (3.3) and (3.4) appropriately, which is being obtained analoguosly as in differential case, after setting $F_{i}=f_{1 i}+c_{1 i} v_{i}$ and $G_{i}=f_{2 i}+\varepsilon^{\mu} c_{2 i} u_{i}$ we will get

$$
\begin{gathered}
\|U\|_{\infty, d} \leq \gamma\left(\varepsilon \sigma_{*}\left\|U_{x, 0}\right\|_{\infty, d}+\left\|c_{1}\right\|_{\infty, d}\|V\|_{\infty, d}+h \sum_{i=1}^{N}\left|f_{1 i}\right|\right) \\
\|V\|_{\infty, d} \leq \\
\leq\left|A_{2}\right|+\left|B_{2}\right|+\alpha_{2}^{-1}\left\|f_{2}\right\|_{\infty, d} \\
+\alpha_{2}^{-1} \varepsilon^{\mu}\left\|c_{2}\right\|_{\infty, d}\|U\|_{\infty, d}
\end{gathered}
$$

The using each of these into another immediately leads to (3.11) and (3.12).

Lemma 3.3 For the truncation errors

$$
\begin{aligned}
R_{1 i} & =f_{1 i}-L_{1}^{h} u\left(x_{i}\right)+c_{1 i} v\left(x_{i}\right), \\
R_{2 i} & =f_{2 i}-L_{2}^{h} v\left(x_{i}\right)+c_{2 i} u\left(x_{i}\right), \\
r= & \left(1+a_{1}(0) \frac{h}{\varepsilon} \sigma_{10}^{(2)}\right)^{-1} \\
& \cdot\left(\varepsilon^{-1} B_{1}-b_{1}(0) A \frac{h}{\varepsilon} \sigma_{10}^{(2)}+\frac{h}{\varepsilon} \sigma_{10}^{(2)} f(0)\right)-u_{x, 0}
\end{aligned}
$$

the following estimates hold

$$
\begin{gathered}
\left|R_{1 i}\right| \leq C h\left(1+h^{-1} \int_{x_{i-1}}^{x_{i+1}}\left|u^{\prime}(x)\right| \mathrm{d} x+h^{-1} \int_{x_{i-1}}^{x_{i+1}}\left|v^{\prime}(x)\right| \mathrm{d} x\right), \\
\left|R_{2 i}\right| \leq C h\left(1+\varepsilon^{\mu}\left\|u^{\prime}(x)\right\|_{\infty,\left[x_{i-1}, x_{i+1}\right]}\right),
\end{gathered}
$$

$$
|r| \leq \frac{C h}{\varepsilon\left(1+a_{1}(0) \sigma_{10} \frac{h}{\varepsilon}\right)}\left(1+\int_{x_{0}}^{x_{1}}\left|u^{\prime}(x)\right| \mathrm{d} x\right) .
$$

Proof. We may write

$$
\begin{aligned}
R_{1 i}= & f_{1 i}-\mathcal{L}_{1}^{h} u\left(x_{i}\right)+c_{1 i} v\left(x_{i}\right) \\
& -h^{-1} \int_{x_{i-1}}^{x_{i+1}}\left[\mathcal{L}_{1} u(x)-c_{1}(x) v(x)-f_{1}(x)\right] \varphi_{i}(x) \mathrm{d} x \\
= & h^{-1} \int_{x_{i-1}}^{x_{i+1}}\left[a_{1}(x)-a_{1}\left(x_{i}\right)\right] u^{\prime}(x) \varphi_{i}(x) \mathrm{d} x \\
& +h^{-1} \int_{x_{i-1}}^{x_{i+1}}\left[b_{1}(x) u(x)-b_{1}\left(x_{i}\right) u\left(x_{i}\right)\right] \varphi_{i}(x) \mathrm{d} x \\
& +h^{-1} \int_{x_{i-1}}^{x_{i+1}}\left[f_{1}\left(x_{i}\right)-f_{1}(x)\right] \varphi_{i}(x) \mathrm{d} x \\
& +h^{-1} \int_{x_{i-1}}^{x_{i+1}}\left[c_{1}\left(x_{i}\right) v\left(x_{i}\right)-c_{1}(x) v(x)\right] \varphi_{i}(x) \mathrm{d} x, \\
R_{2 i}= & f_{2 i}-\mathcal{L}_{2}^{h} v\left(x_{i}\right)+\varepsilon^{\mu} c_{2 i} u\left(x_{i}\right) \\
& -\lambda_{i} h^{-1} \int_{x_{i-1}}^{x_{i+1}}\left[\mathcal{L}_{2} v-\varepsilon^{\mu} c_{2}(x) u(x)-f_{2}(x)\right] \psi_{i}(x) \mathrm{d} x \\
\equiv & h^{-1} \lambda_{i} \int_{x_{i-1}}^{x_{i+1}}\left[a_{2}(x)-a_{2}\left(x_{i}\right)\right] v(x) \psi_{i}(x) \mathrm{d} x \\
& +\lambda_{i} h^{-1} \int_{x_{i-1}}^{x_{i+1}}\left[f_{2}\left(x_{i}\right)-f_{2}(x)\right] \psi_{i}(x) \mathrm{d} x \\
& +h^{-1} \lambda_{i} \varepsilon^{\mu} \int_{x_{i-1}}^{x_{i+1}}\left[c_{2}\left(x_{i}\right) u\left(x_{i}\right)-c_{2}(x) u(x)\right] \psi_{i}(x) \mathrm{d} x, \\
&
\end{aligned}
$$

$$
\begin{aligned}
r= & \frac{1}{\varepsilon\left(1+a_{1}(0) \frac{h}{\varepsilon} \sigma_{10}^{(2)}\right)} \\
& \cdot \int_{x_{0}}^{x_{1}}[b(x) u(x)-b(0) u(0)] \varphi_{0}^{(2)}(x) \mathrm{d} x \\
& +\int_{x_{0}}^{x_{1}}\left[a_{1}(x)-a_{1}(0)\right] u^{\prime}(x) \varphi_{0}^{(2)}(x) \mathrm{d} x \\
& -\int_{x_{0}}^{x_{1}} c_{1}(x) v(x) \varphi_{0}^{(2)}(x) \mathrm{d} x x \\
& -\int_{x_{0}}^{x_{1}}\left[f_{1}(x)-f_{1}(0)\right] \varphi_{0}^{(2)}(x) \mathrm{d} x,
\end{aligned}
$$

where 


$$
\begin{aligned}
& \varphi_{i}(x)= \begin{cases}\varphi_{i}^{(1)}(x)=\frac{e^{\frac{a_{1}\left(x_{i}\right)}{\varepsilon}\left(x-x_{i-1}\right)}-1}{e^{\frac{a_{1}\left(x_{i}\right) h}{\varepsilon}}-1}, & x_{i-1}<x<x_{i}, \\
\varphi_{i}^{(2)}(x)=\frac{1-e^{-a_{1}\left(x_{i}\right)\left(x_{i+1}-x\right) / \varepsilon}}{1-e^{-a_{1}\left(x_{i}\right) h / \varepsilon},} & x_{i}<x<x_{i+1}, \\
0, & x \notin\left(x_{i-1}, x_{i+1}\right),\end{cases} \\
& \psi_{i}(x) \\
& = \begin{cases}\psi_{i}^{(1)}(x)=\frac{\sinh \sqrt{a_{2}\left(x_{i}\right) / \varepsilon}\left(x-x_{i-1}\right)}{\sinh \sqrt{a_{2}\left(x_{i}\right) / \varepsilon},}, & x_{i-1}<x<x_{i}, \\
\psi_{i}^{(2)}(x)=\frac{\sinh \sqrt{a_{2}\left(x_{i}\right) / \varepsilon}\left(x_{i+1}-x\right)}{\sinh \sqrt{a_{2}\left(x_{i}\right) / \varepsilon} h}, & x_{i}<x<x_{i+1}, \\
0, & x \notin\left(x_{i-1}, x_{i+1}\right),\end{cases} \\
& \lambda_{i}=\left(\begin{array}{ll}
\left.h^{-1} \int_{x_{i-1}}^{x_{i+1}} \psi_{i}(x) \mathrm{d} x\right)^{-1}=\frac{h \sqrt{a_{2}\left(x_{i}\right) / \varepsilon}}{2 \tanh \sqrt{a_{2}\left(x_{i}\right) / \varepsilon} h} .
\end{array}\right.
\end{aligned}
$$

We note that $\varphi_{i}^{(1)}, \varphi_{i}^{(2)}$ and $\psi_{i}^{(1)}, \psi_{i}^{(2)}$ are the solutions of following problems respectively:

$$
\begin{gathered}
-\varepsilon \psi^{\prime \prime}(x)+a_{2}(x) \psi(x)=0, x_{i-1}<x<x_{i} \\
\psi\left(x_{i-1}\right)=0, \psi\left(x_{i}\right)=1, \\
-\varepsilon \psi^{\prime}(x)+a_{2}(x) \psi(x)=0, x_{i}<x<x_{i+1} \\
\psi\left(x_{i}\right)=1, \psi\left(x_{i+1}\right)=0 .
\end{gathered}
$$

The relations (3.18)-(3.20), by using also the above properties of $\varphi(x)$ and $\psi(x)$ leads immediately to (3.15)-(3.17).

Theorem 3.1 Let $a_{k}(x), b_{k}(x), c_{k}(x)$, $f_{1}(x) \in C[0,1], f_{2}(x) \in C^{1}[0,1], \mu \geq 1,(k=1,2)$. Then the solution of the difference problem (3.1)-(3.4) converges uniformly in $\varepsilon$ to the solution of (1.1)-(1.4) with rate $O(h)$.

Proof. Let $z_{1 i}=U_{i}-u_{i}, \quad z_{2 i}=V_{i}-v_{i}$. Then for the errors of the approximate solution

$$
z_{k i}(k=1,2 ; i=0,1,2, \cdots, N) \text { we have }
$$

$$
\begin{gathered}
\mathcal{L}_{1}^{h} z_{1 i}=c_{1 i} z_{2 i}+R_{1 i}, i=1,2, \cdots, N-1, \\
\mathcal{L}_{2}^{h} z_{2 i}=\varepsilon^{\mu} c_{2 i} z_{1 i}+R_{2 i}, i=1,2, \cdots, N-1, \\
z_{10}=0, z_{1 x, 0}=r_{0}, \\
z_{20}=z_{2 N}=0,
\end{gathered}
$$

where $R_{1 i}, R_{2 i}, r_{0}$ are approximating errors from Lemma
3.3. Using Lemma 3.2, we obtain:

$$
\left\|z_{1}\right\|_{\infty, d},\left\|z_{2}\right\|_{\infty, d} \leq C\left(\varepsilon \sigma_{*}\left|r_{0}\right|+h \sum_{i=1}^{N}\left|R_{1 i}\right|+\left\|R_{2}\right\|_{\infty, d}\right) .
$$

By virtue that of (3.15)-(3.17) all terms in right-hand side of this inequality have the rate $O(h)$ and hence the proof follows immediately.

\section{Numerical Example}

Consider the particular problem with

$$
\begin{gathered}
a_{1}=x+2, b_{1}=e^{-x} / 2, c_{1}=\sin \pi x, f_{1}=x^{2}, \\
a_{2}=e^{x+1}, c_{2}=x / 2, f_{2}=\sqrt{x+1}, \mu=1, \\
A_{1}=B_{1}=1, A_{2}=-1, B_{2}=0 .
\end{gathered}
$$

The initial guess is chosen as

$$
V_{i}^{(0)}=x_{i}-1
$$

and stopping criterion is

$$
\begin{aligned}
& \left|U^{(n)}-U^{(n-1)}\right|<10^{-5} \\
& \left|V^{(n)}-V^{(n-1)}\right|<10^{-5} .
\end{aligned}
$$

We calculate an experimental rates of convergence $p_{k}(k=1,2)$ using double mesh method as follows [4,5]:

$$
p_{k}^{\varepsilon, h}=\ln \left(e_{k}^{\varepsilon, h} / e_{k}^{\varepsilon, h / 2}\right) / \ln 2,
$$

where

$$
\begin{aligned}
e_{1}^{\varepsilon, h} & =\max _{i}\left|U_{i}^{\varepsilon, h}-U_{i}^{\varepsilon, h / 2}\right|, \\
e_{2}^{\varepsilon, h} & =\max _{i}\left|V_{i}^{\varepsilon, h}-V_{i}^{\varepsilon, h / 2}\right| .
\end{aligned}
$$

The convergence is uniform, i.e., rate of convergence independenty of perturbation parameter. Some obtained values for

$$
p_{k}^{h}=\max _{\varepsilon} p_{k}^{\varepsilon, h}\left(\varepsilon=0.5,10^{-2}, 10^{-4}, 10^{-6}\right)
$$

are listed in the table

\begin{tabular}{cccc}
\hline$h$ & 0.1 & 0.05 & 0.025 \\
$p_{1}, p_{2}$ & $1.61,1.58$ & $1.32,1.26$ & $1.01,0.99$ \\
\hline
\end{tabular}

\section{Conclusion}

The singularly perturbed initial-boundary value problem for a linear second order differential system is considered. To solve this problem, an exponentially fitted difference scheme on a uniform mesh is presented. First order convergence in the discrete maximum norm, independently of the perturbation parameter is obtained. Obtained in numerical example experimental rates of convergence in 
agreement with theoretical values.

\section{Acknowledgements}

The author is grateful to the anonymous referees for his comments and suggestions which helped improve the quality of manuscript.

\section{REFERENCES}

[1] R. E. O'Malley, "Singular Perturbations Methods for Ordinary Differential Equations," Springer Verlag, New York, 1991. doi:10.1007/978-1-4612-0977-5

[2] A. H. Nayfeh, "Introduction to Perturbation Techniques," Wiley, New York, 1993.

[3] A. M. Ilin, "A Difference Scheme for a Differential Equation with a Small Parameter Affecting the Highest Derivative," Matematicheskie Zametki, Vol. 6, 1969, pp. 237-248 (Russian).

[4] E. R. Doolan, J. J. H. Miller and W. H. A. Schilders, "Uniform Numerical Methods for Problems with Initial and Boundary Layers," Boole Press, Dublin, 1980.

[5] P. A. Farrell, A. F. Hegarty, J. J. H. Miller, E. O'Riordan and G. I. Shishkin, "Robust Computational Techniques for Boundary Layers," Chapman-Hall/CRC, New York, 2000.

[6] H. G. Roos, M. Stynes and L. Tobiska, "Robust Numerical Methods for Singularly Perturbed Differential Equations, Convection Diffusion and Flow Problems," Springer-Verlag, Berlin, Heidelberg, 2008.

[7] T. Linss, "Layer-Adapted Meshes for Reaction-Convection-Diffusion Problems, Lecture Notes in Mathematics," Springer, Heidelberg, 1985.
[8] G. M. Amiraliyev and H. Duru, "A Uniformly Convergent Finite Difference Method for an Initial Value Problem," Applied Mathematics and Mechanics, Vol. 20, No. 4, 1999, pp. 363-370. doi:10.1007/BF02458564

[9] G. M. Amiraliyev, "The Convergence of a Finite Difference Method on Layer-Adapted Mesh for a Singularly Perturbed System," Applied Mathematics and Computation, Vol. 162, No. 3, 2005, pp. 1023-1034. doi:10.1016/j.amc.2004.01.015

[10] S. Natesan and B. S. Deb, "A Robust Computational Method for Singularly Perturbed Coupled System of Reaction-Diffusion Boundary-Value Problems," Applied Mathematics and Computation, Vol. 188, No. 1, 2007, pp. 353-364. doi:10.1016/j.amc.2006.09.120

[11] S. Hemavathi, T. Bhuvaneswari, S. Valarmathi and J. J. H. Miller, "A Parameter Uniform Numerical Method for a System of Singularly Perturbed Ordinary Differential Equations," Applied Mathematics and Computation, Vol. 191, No. 1, 2007, pp. 1-11. doi:10.1016/j.amc.2006.05.218

[12] T. Linss and M. Stynes, "Numerical Solution of Systems of Singularly Perturbed Differential Equations," Computational Methods in Applied Mathematics, Vol. 9, No. 2, 2009, pp. 165-191.

[13] T. Linss, "Analysis of a System of Singularly Perturbed Convection-Diffusion Equations with Strong Coupling," SIAM Journal on Numerical Analysis, Vol. 47, No. 3, 2009, pp. 1847-1862. doi:10.1137/070683970

[14] Z. D. Cen, A. M. Xu and A. Le, "A Second-Order Hybrid Finite Difference Scheme for a System of Singularly Perturbed Initial Value Problems," Journal of Computational and Applied Mathematics, Vol. 234, No. 12, 2010, pp. 3445-3457. doi:10.1016/i.cam.2010.05.006 